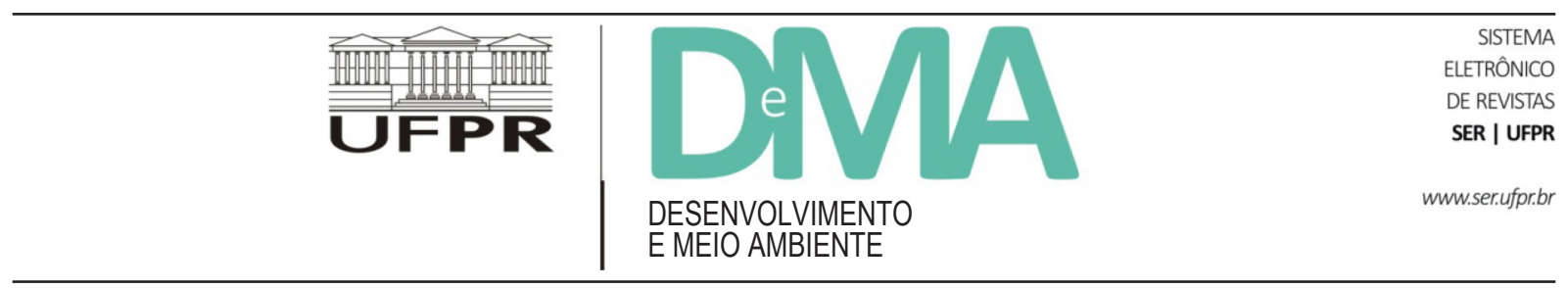

\title{
Delineamento da produção científica sobre desastres no Brasil no início deste século
}

\section{An Overview of Brazilian Scientific Production on Disasters in the Beginning of this Century}

\author{
Angela Cassia RODRIGUES ${ }^{1 *}$, Wanda Maria Risso GÜNTHER ${ }^{1}$, Maria da Penha VASCONCELLOS ${ }^{1}$, Gabriela \\ Marques Di GIULIO ${ }^{1}$, Maria Eugenia Gimenez BOSCOV ${ }^{2}$ \\ ${ }^{1}$ Faculdade de Saúde Pública, Universidade de São Paulo (USP), São Paulo, SP, Brasil. \\ ${ }^{2}$ Escola Politécnica, Universidade de São Paulo (USP), São Paulo, SP, Brasil. \\ *E-mail de contato: angelacassia@usp.br
}

Artigo recebido em 15 de dezembro de 2014, versão final aceita em 1 de julho de 2015.

RESUMO

ABSTRACT

Este artigo busca contribuir para a compreensão de como vem se delineando a produção científica brasileira sobre desastres no início do século XXI, a partir de levantamento de três tipos de publicações: i) teses e dissertações; ii) artigos em periódicos brasileiros indexados na base SciELO; e iii) artigos em periódicos indexados na base de dados Web of Science que tenha um ou mais pesquisadores brasileiros como autores. Os resultados indicam as principais áreas do conhecimento que têm se dedicado à realização de pesquisas sobre o tema, a produção acadêmica em formato de teses e dissertações e as publicações em periódicos nacionais e internacionais com autores e/ou coautores brasileiros. Evidencia alguns aspectos envolvidos no crescimento da produção científica brasileira sobre desastres a partir de 2008, abordando os principais temas das investigações e possíveis tendências nesse campo.

Palavras-chave: desastres; produção científica; interdisciplinaridade; Brasil.

In this article we seek to present an overview of Brazilian scientific production on disasters in the beginning of this century based on a review of three data bases: (i) PhD Thesis and Master Dissertations; (ii) articles in Brazilian journals indexed in SciELO database; and (iii) articles indexed in Web of Science database which have Brazilian researchers among the authors. The results point out the main scientific areas, and $\mathrm{PhD}$ Thesis and Master Dissertations which have focused this subject. Our results also bring forward the published papers in national and international journals which are focused on disaster issue and have been produced by Brazilian authors. We also seek to debate some aspects related to the increase of Brazilian scientific production on disasters after 2008, and to point out possible trends on this field.

Keywords: disasters; scientific production; interdisciplinarity; Brazil. 


\section{Introdução}

Em âmbito mundial, diversas áreas do conhecimento vêm se dedicando ao estudo dos desastres e seus aspectos. Na perspectiva histórica, Lindell (2011) aponta que a primeira pesquisa científica sistemática sobre desastres data de 1920 e tratou de estudo sociológico de Samuel Prince sobre a explosão de um navio de carga em Halifax, Canadá, em 1917. O primeiro tratado sobre desastres foi publicado pelo sociólogo russo Pitirim Sorokin, em 1942, e considerava quatro tipos de desastres, correspondentes a grandes convulsões coletivas, com certa densidade no tempo histórico e extensão considerável no espaço geossocial: fome, epidemias, guerras e revoluções (Martins, 2011, p. 255). Martins (2011) ressalta ainda que a omissão dos desastres naturais nessa tipologia proposta por Sorokin se deve, em parte, por tais eventos serem mais localizados no tempo e no espaço, como erupções vulcânicas, ou difusas, como alterações climáticas.

Em 1963 foi criado o primeiro centro de pesquisa social do mundo a se dedicar ao estudo dos desastres, o Disaster Research Center (DRC), na Universidade do Estado de Ohio (EUA), o qual, em 2010, computava cerca de 700 pesquisas empíricas (Kendra et al., 2011).

Um dos grandes desafios na área de estudos dos desastres relaciona-se a sua própria conceituação, caracterizada pela grande diversidade e pela inexistência de consenso (Valêncio, 2010).

Na perspectiva das ciências ambientais e da saúde pública, a ocorrência de fenômenos da natureza ou de acidentes devido a falhas humanas na utilização de tecnologias somente se configura como desastre caso haja a exposição de populações humanas. Os fenômenos citados são considerados eventos detonadores e conceituados como perigos ou ameaças aos quais as populações encontram-se expostas (Freitas et al., 2012).

A Estratégia Internacional de Redução de Desastres da ONU (UNISDR) define desastre como "uma séria interrupção no funcionamento de uma comunidade ou sociedade que ocasiona grande quantidade de mortes, perdas e impactos materiais, econômicos e ambientais que excedem a capacidade da comunidade ou sociedade afetada para enfrentar a situação, mediante uso de seus próprios recursos" (ISDR, 2009). Essa definição admite que o momento exato para um evento adverso ser considerado desastre é quando se denota a incapacidade local de enfrentamento da situação pós-evento, decorrente de perdas humanas e materiais que interrompem a funcionalidade da comunidade ou sociedade considerada. Nesse conceito fica implícito que um mesmo evento pode representar desastre para uma comunidade, e não para outra que possua maior capacidade de enfrentamento ou resposta.

Já no âmbito brasileiro, tomando como referência a Política Nacional de Defesa Civil, o termo desastre se refere ao "resultado de eventos adversos, naturais ou provocados pelo homem, sobre um ecossistema vulnerável, causando danos humanos, materiais e/ou ambientais e consequentes prejuízos econômicos e sociais" (Brasil, 2012a). Essa definição induz à ideia de danos; logo, desastre é o resultado (danos de diversas naturezas) que ocasiona prejuízos econômicos e sociais a ecossistemas vulneráveis.

Em 2008, foi publicada a norma denominada $D i-$ saster Loss Database Standards, resultante de trabalho envolvendo especialistas do Centre for Research on the Epidemiology of Disasters (CRED), das companhias de seguro Munich Re., Swiss Re e Asian Disaster Reduction Center (CCRA) e do Programa das Nações Unidas para o Desenvolvimento (PNUD) (GRIP, 2008; Wirtz et al., 2014). Esse documento aponta o problema da multiplicidade de definições do termo e conceitua "evento de desastre" como fenômeno, natural ou não, que causa ruptura no funcionamento de uma comunidade ou de uma sociedade, ou efeitos negativos sobre a vida humana, a saúde e/ou sobre a infraestrutura social, ambiental, material ou econômica (GRIP, 2008, tradução livre). Nota-se que essa definição, de forma semelhante à da Defesa Civil, não utiliza os termos/condições constantes da definição da UNISDR, "exceder a capacidade de uma comunidade para lidar com os efeitos" ou "a necessidade de ajuda externa".

As classificações mais tradicionais na área de gestão de desastres os diferenciam, quanto a sua origem ou causa, em dois grupos: "desastres naturais", aqueles que têm como causa um fenômeno natural, geralmente de grande intensidade, agravado ou não pela atividade humana, como chuvas intensas, ventos fortes, tremores 
de terra; e "desastres humanos ou antropogênicos", que resultam de ações ou omissões humanas, estando relacionados com as atividades do homem, como agente ou autor" (Tominaga et al., 2009). Como exemplos de desastres antropogênicos, também denominados desastres tecnológicos, podem ser citados acidentes aéreos, incêndios urbanos, contaminação do ambiente por substâncias perigosas, rompimento de barragens, entre outros.

Em 2006, pesquisadores do CRED e do Munich RE Risks Research conduziram um estudo com o objetivo de padronizar a terminologia e a classificação dos desastres entre os principais bancos de dados internacionais (Below, 2009). Em decorrência desse estudo, a classificação utilizada atualmente pelo International Disaster Database (EM-DAT), um dos principais bancos de dados de desastres criado e administrado pelo CRED, distingue dois grupos genéricos: desastres naturais e desastres tecnológicos. O primeiro grupo está dividido em cinco subgrupos: biológicos, geofísicos, hidrológicos, meteorológicos e climatológicos, enquanto que o segundo em três: acidentes industriais, de transporte e acidentes diversos (colapsos de estruturas não industriais, explosões, incêndios).

Em 2012, para atender à classificação dos desastres do EM-DAT, a Secretaria Nacional de Proteção e Defesa Civil (SEDEC), por meio da Instrução Normativa $n^{\circ} 1$, de 24 de agosto de 2012, passou a adotar a Codificação Brasileira de Desastres (COBRADE) (Brasil, 2012b).

\subsection{A ênfase nos desastres naturais}

Devido às alterações climáticas observadas nas últimas décadas e ao consequente aumento da frequência e da intensidade de fenômenos naturais extremos de origem hidrometeorológica e climatológica (IPCC, 2007; 2014), verifica-se, tanto na literatura especializada quanto em relatórios institucionais, maior ênfase aos desastres naturais.

Diversos autores têm apresentado perspectiva de análise dos desastres naturais que extrapola a visão tecnocrática, reconhecendo a vulnerabilidade social como importante fator na produção de risco e impactos. Essa perspectiva considera que, mesmo tendo como eventos detonadores fenômenos da dinâmica da natureza, os desastres não são naturais, mas, sim, uma interação entre ambiente e ações sociais, em que fatores históricos, políticos e econômicos estão presentes em contextos complexos (Ribeiro, 1995; Valêncio, 2010; Soriano et al., 2013; Carmo, 2014).

Valêncio (2014) aponta que, na América Latina, os órgãos de proteção e defesa civil se referem aos grandes impactos humanos e materiais associados aos fenômenos da natureza (chuvas intensas, secas, vendavais) como desastres naturais. Para a autora, esse enfoque visa evitar um questionamento aprofundado sobre o processo sócio-histórico dentro do qual se desenvolve a dinâmica socioespacial, e nesse processo estão os fatores que explicariam os diferentes níveis de danos e prejuízos entres comunidades expostas a eventos semelhantes.

Outra questão que ganha destaque é o acelerado processo de urbanização, uma vez que atualmente mais da metade da população mundial $(50,9 \%)$ se estabelece nas cidades (United Nations, 2012). A combinação de crescimento populacional com expansão da urbanização aumenta o potencial de risco de ocorrência de desastres, tanto naturais quanto tecnológicos, para todas as comunidades, em especial nas cidades onde há maior densidade populacional (IPCC, 2014).

\subsection{Brasil: ocorrências de desastres e a produção científica}

Levantamento realizado no banco internacional de dados de desastres EM-DAT, em 20 de outubro de 2014, indicou o registro de 77 desastres naturais ocorridos no Brasil, no período de 2000 a 2013, os quais resultaram em 2.830 mortes, 1.673 feridos e 10 milhões de pessoas afetadas. No mesmo período, foi encontrado registro de 68 desastres tecnológicos ocorridos no Brasil, que levaram a 2.047 mortes, 2.182 feridos e 552 mil pessoas afetadas (EM-DAT, 2014). Enquanto pode ser considerada certa proporcionalidade entre mortos e feridos em ambos os tipos de desastres, nota-se que os desastres naturais afetam, consideravelmente, maior número de pessoas.

O Atlas Brasileiro de Desastres Naturais - 1991 a 2010, elaborado pelo Centro Universitário de Estudos 
e Pesquisas sobre Desastres (CEPED), da Universidade Federal de Santa Catarina, aponta que os números do EM-DAT estão muito aquém da realidade. A metodologia utilizada nesse estudo envolveu resgate histórico dos registros de ocorrência de desastres por meio de levantamento de documentos oficiais existentes em órgãos federais, estaduais e municipais de Defesa Civil do país. Foram levantados dados do período de 1991 a 2010. No período de 2000 a 2010, há registro de ocorrência de 23.238 desastres naturais, ou seja, mais de 300 vezes o registrado pelo EM-DAT. Esse banco de dados do histórico dos desastres brasileiros associados a fenômenos naturais indicou que os eventos de estiagem, seca, inundação brusca e alagamento são os mais recorrentes no país (CEPED UFSC, 2012).

Em que pese o significativo aumento das ocorrências de desastres no Brasil, o debate sobre o tema ainda é recente. Um dos primeiros grupos de estudo envolvidos com a temática foi criado na Universidade Federal de São Carlos (UFSCar), em 2003, voltando-se à área da Sociologia dos Desastres ${ }^{1}$. Intitulado inicialmente Grupo de Estudos e Pesquisas em Desastres (GEPED), em 2005 institucionalizou-se junto ao Departamento de Ciências Sociais (DCSo) como Núcleo de Estudos e Pesquisas Sociais em Desastres (NEPED). O grupo tem se destacado, sobretudo, por suas publicações, a partir de 2009, abordando o tema dos desastres.

$\mathrm{Na}$ tentativa de mapear os grupos de pesquisa cadastrados no Diretório dos Grupos de Pesquisa (DGP) do Conselho Nacional de Desenvolvimento Científico e Tecnológico (CNPq), foi realizada busca em 14 de novembro de 2014, utilizando o termo "desastre" no nome do grupo, sendo considerados como resultados aqueles constituídos entre os anos de 2000 e 2013 (Tabela 1). Foram encontrados 17 grupos, a maioria (12) constituída a partir de 2008. Observa-se a predominância das áreas das Geociências (41\%) e Engenharias (29\%). Foram identificados também outros 36 grupos de pesquisa, de 15 diferentes áreas de conhecimento, que, embora não possuam o termo "desastre" no nome do grupo, utilizam-no para identificar uma linha de pesquisa. Destes, sete (19\%) pertencem à área das Engenharias, e dez (28\%) são de Geociências, verificando-se a mesma predominância do campo de atuação observada anteriormente (Figura 1).

TABELA 1 - Grupos de pesquisa identificados no diretório do CNPq, por ano de criação e área, no período de 2000 a 2013.

\begin{tabular}{clc}
\hline $\begin{array}{c}\text { Ano de } \\
\text { Constituição }\end{array}$ & \multicolumn{1}{c}{ Grupo } & Área \\
\hline 2000 & Centro Universitário de Estudos e Pesquisas sobre Desastres (CEPED UFSC) & Engenharias \\
2003 & Grupo de Estudos de Desastres Socionaturais - GEDN & Geociências \\
2004 & Grupo de Estudo e Pesquisa dos Desastres Naturais - GEDEN & Engenharias \\
2007 & Geotecnologias para desastres naturais & Geociências \\
2007 & Gestão de risco e desastres relacionados a eventos naturais & Geociências \\
2008 & Estudos em Vulnerabilidades e Desastres & Geociências \\
2010 & Grupo de Estudo e Pesquisa sobre Desastres na Amazônia - PEGEDAM & Geografia \\
2010 & Gestão de Riscos de Desastres - GRID & Engenharias \\
2010 & Núcleo de Pesquisa sobre Desastres - NUPED & Geografia \\
\hline
\end{tabular}

\footnotetext{
${ }^{1}$ A Sociologia dos Desastres é uma subárea da Sociologia do Desenvolvimento que, segundo Valêncio (2009), encontra-se em franco crescimento nas principais arenas científicas internacionais.
} 


\begin{tabular}{clc}
\hline $\begin{array}{c}\text { Ano de } \\
\text { Constituição }\end{array}$ & \multicolumn{1}{c}{ Grupo } & Área \\
\hline 2010 & Núcleo de Pesquisas em Desastres Naturais & Engenharias \\
2011 & Clima e Desastres Naturais & Geociências \\
2011 & Desastres Naturais e Sustentabilidade Ambiental & Geociências \\
2011 & Grupo Interdisciplinar de Atenção às Situações de Desastres & Enfermagem \\
2011 & Sensoriamento remoto no monitoramento de mudanças climáticas e desastres na- & Geociências \\
2012 & turais & Geografia \\
2012 & Gestão de Riscos de Desastres & Saúde Cole- \\
& Vulnerabilidade Socioambiental, Desastres e Saúde & Engenharias \\
\hline
\end{tabular}

FONTE: Elaboração das autoras.

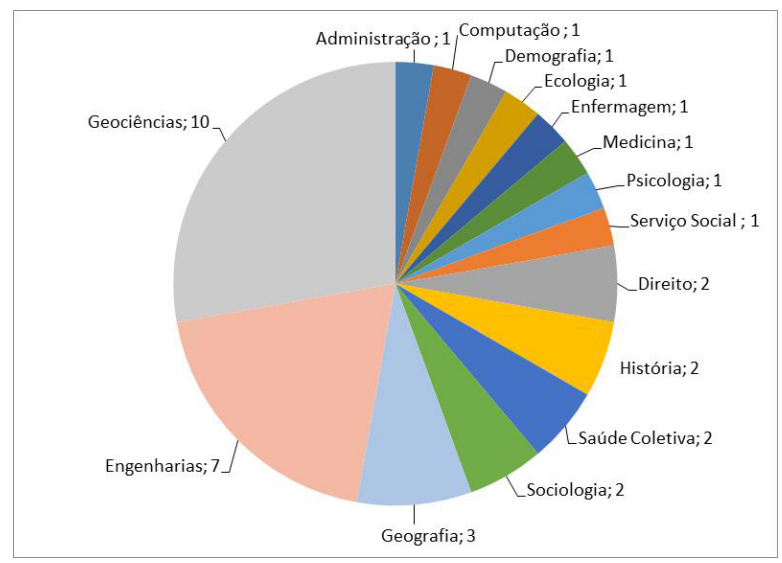

FIGURA 1 - Grupos com linha de pesquisa contendo o termo "desastre" na identificação.

FONTE: Elaboração das autoras.

A relevância da questão dos desastres se evidencia tanto no âmbito global quanto no nacional. Em âmbito internacional, as publicações em periódicos sobre o tema desastres naturais e desastres tecnológicos, disponibilizados na base de dados Web of Science (WoS), se considerados somente os artigos e revisões, ultrapassam a marca de 5,4 mil publicações, no período de 2000 a $2013 .^{2}$

Dentro das estratégias globais para o enfrentamento dos episódios de desastres, em 2005 foi assinado por 168 países o Protocolo de Hyogo, cujo objetivo é fortalecer e aumentar a capacidade de resiliência a situações de eventos adversos, de nações e comunidades com potencial de risco de ocorrência de desastres (UNISDR, 2005). O documento estabelece cinco ações prioritárias e define metas a serem alcançadas até 2015. As ações definidas no Marco de Hyogo incluem:

- Garantir que a redução de risco de desastres (RRD) seja uma prioridade nacional e local, com sólida base institucional para sua implantação;

- Identificar, avaliar e observar de perto os riscos dos desastres e melhorar os alertas prévios;

- Utilizar o conhecimento, a inovação e a educação para criar uma cultura de segurança e resiliência em todos os níveis;

\footnotetext{
${ }^{2}$ Levantamento efetuado em 22 de setembro de 2014. Foram realizadas duas buscas: a primeira utilizando o termo "Natural Disaster*”, e a segunda, "Technological Disaster*".
} 
- Reduzir os fatores fundamentais de risco;

- Fortalecer a preparação para desastres para uma resposta eficaz em todos os níveis.

O encaminhamento dessas ações requer a criação de competências nacionais e regionais, na qual a pesquisa científica no Brasil tem papel importante a desempenhar. Nesse sentido, citam-se como forma ilustrativa alguns encaminhamentos recentes, na perspectiva do fortalecimento da pesquisa científica no âmbito brasileiro.

A Coordenação de Aperfeiçoamento de Pessoal de Nível Superior (CAPES), por exemplo, lançou o Edital Pró-Alertas no 24/2014 - Gestão de Desastres Naturais, com o objetivo de fomentar e ampliar a produção científica, pesquisas básicas e aplicadas para o aprofundamento do conhecimento sobre o tema. Leitura mais atenta do edital indica também maior ênfase nas áreas temáticas pertencentes aos campos das Ciências Exatas e da Terra e Engenharias.

Ainda seguindo essa premissa, o Centro Nacional de Monitoramento e Alertas de Desastres Naturais (CEMADEN), vinculado à Secretaria de Políticas e Programas de Pesquisas e Desenvolvimento (SEPED), do Ministério da Ciência, Tecnologia e Inovação (MCTI), lançou em 2013 um edital para a seleção de pesquisadores para atuação na área.

Devido à relevância e à atualidade do tema, o periódico Ciência e Saúde Coletiva publicou no mês de setembro de 2014 o número temático "Desastres naturais - impactos, vulnerabilidades e organização do setor saúde", apresentando 13 artigos sobre os efeitos dos desastres naturais em pessoas e ambiente. O periódico Ambiente e Sociedade também fez uma chamada para a publicação de um número especial, "Desastres Naturais e Socioambientais", em 2014, apresentando 16 artigos.

Na vertente de incremento às instituições acadêmicas, a CAPES credenciou e autorizou no ano de 2014 a abertura de três mestrados profissionais voltados à gestão de riscos e desastres naturais nas Universidades Federais do Pará, de Pernambuco e de Santa Catarina.
A partir desses levantamentos iniciais e considerando-se a pertinência do tema, este artigo tem por objetivos apresentar um delineamento da produção científica brasileira sobre desastres a partir do século XXI, apontar os principais temas abordados nas investigações e identificar algumas tendências nesse campo.

\section{Métodos}

O universo do estudo incluiu os seguintes levantamentos:

a. Teses e dissertações defendidas até 2013, disponibilizadas no Banco de Teses e Dissertações da CAPES, no portal Domínio Público e na Biblioteca Digital Brasileira de Teses e Dissertações (BDTD), contendo uma das seguintes palavras-chave: "desastre", "desastres" ou "desastres naturais". Com o objetivo de capturar o maior número possível de trabalhos, não foi fixado um marco temporal inicial para esse levantamento, entretanto as teses levantadas são posteriores ao ano 2000. Foram identificados 90 trabalhos, classificados segundo as variáveis utilizadas para análise: i) ano de defesa da dissertação ou tese; e ii) área de conhecimento na qual a dissertação ou tese foi registrada. Para o enquadramento e a classificação dos trabalhos, adotou-se a Tabela de Áreas de Conhecimento da CAPES, até seu terceiro nível de especificação;

b. Todos os artigos disponibilizados na base de dados de livre acesso SciELO Brasil, publicados até 2013, contendo as palavras-chave "desastre", "desastres", ou "desastres naturais", que têm pesquisadores brasileiros como um dos autores. O objetivo foi capturar o maior número de artigos que abordassem o tema dos desastres, publicados nos periódicos científicos brasileiros. A escolha da base SciELO Brasil deveu-se ao fato de que sua coleção é composta apenas por periódicos científicos publicados

\footnotetext{
${ }^{3}$ A base SciELO conta com 344 periódicos das seguintes áreas: Engenharias, Linguística, Letras e Artes, Ciências Agrárias, Ciências Biológicas, Ciências da Saúde, Ciências Exatas e da Terra, Ciências Humanas e Ciências Sociais Aplicadas (consulta realizada em 30 de dezembro de 2014 no endereço http://www.scielo.br).
} 
no Brasil, ${ }^{3}$ com avaliação dos artigos por pares, baseada em critérios e procedimentos adotados internacionalmente pelas bases de dados. Não foi fixado marco temporal inicial para esse levantamento, entretanto as publicações levantadas são posteriores ao ano 2000. Foram identificados 28 artigos, e, após a leitura dos resumos, um foi excluído por não ter nenhuma relação com o estudo, restando 27 trabalhos. As variáveis utilizadas para a análise desses trabalhos foram: i) ano de publicação; ii) tipo de desastre abordado; e iii) área de conhecimento em que se enquadra, a partir da leitura dos resumos e de acordo com as áreas de conhecimento da tabela CAPES para teses e dissertações.

c. Todos os artigos ou revisões encontrados na base de dados Web of Science (WoS), publicados no período de 2000 a 2013, utilizando-se como critério de busca as palavras "disaster*" e "Brazil", e que têm ao menos um pesquisador brasileiro entre os autores. Foram utilizados dois filtros, um para os tipos de documentos "artigos" e "revisões" e outro para a base de dados Web of Science Core Collection. A busca, realizada em setembro de 2014, identificou 49 publicações. O objetivo foi capturar as publicações em periódicos internacionais que abordassem a temática dos desastres com ocorrências no Brasil. Essa base de dados foi adotada por ser multidisciplinar e contar atualmente com mais de 9.000 periódicos indexados. Após a leitura dos resumos desses artigos, foram excluídas as publicações em periódicos científicos brasileiros, constantes da base de dados SciELO Brasil e já identificadas previamente na busca do item (ii); publicações não relacionadas à temática desastres (considerando as definições tradicionais) e publicações exclusivamente de autores não brasileiros. Resultaram 13 publicações. As variáveis utilizadas para análise desses trabalhos foram: i) ano de publicação; ii) tipo de desastre abordado; e iii) área de pesquisa a que se refere, de acordo com a classificação da base de dados WoS.

\section{Resultados}

Os resultados do levantamento efetuado são apresentados na sequência, subdivididos em três tópicos: i) teses e dissertações; ii) artigos em periódicos nacionais; e iii) artigos em periódicos internacionais. A Figura 2 apresenta a produção temporal dos três primeiros tópicos, em quantidade de publicações em periódicos e trabalhos acadêmicos, no período de 2000 a 2013.

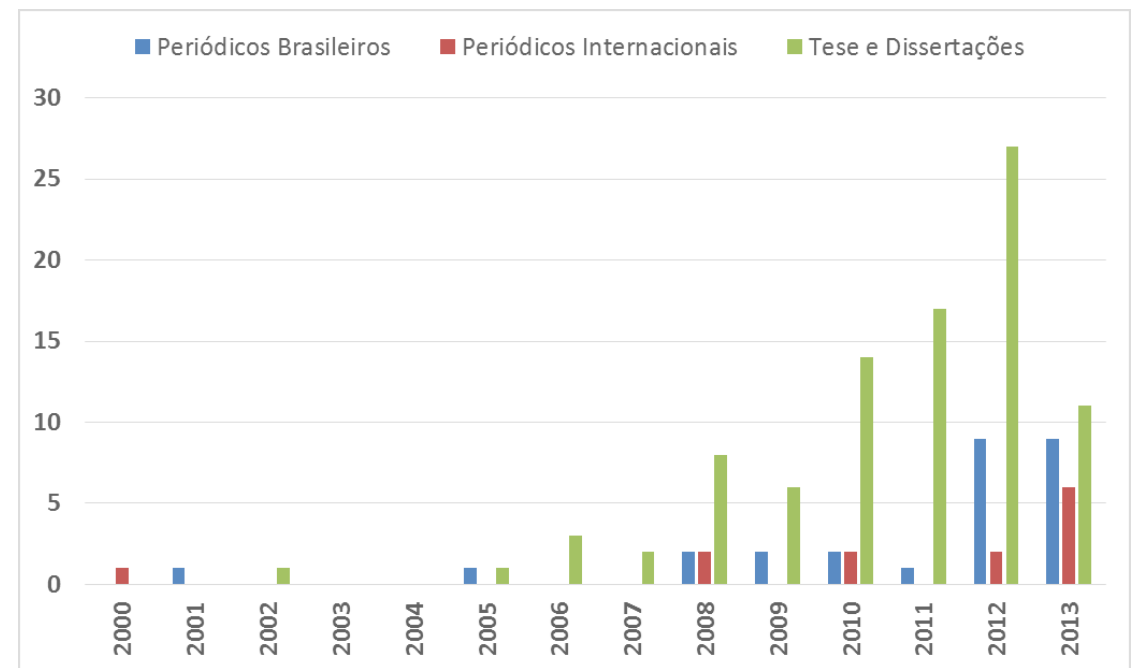

FIGURA 2 - Distribuição da produção científica sobre desastre no Brasil de 2000 a 2013.

FONTE: Elaboração das autoras. 


\subsection{Teses e dissertações}

No período considerado, foi encontrado o total de 90 trabalhos, sendo 16 (18\%) teses de doutorado e 74 (82\%) dissertações de mestrado. O maior número de trabalhos (41) foi encontrado no Banco de Teses da CAPES, que só possui cadastradas as teses e dissertações dos anos de 2011 e 2012 em função da reestruturação do banco. Na época da coleta dos dados, a equipe da CAPES responsável por essa base realizava análise dos dados informados para identificar registros não incompletos. Por essa razão, optou-se pelo levantamento complementar no portal Domínio Público e na Biblioteca Digital Brasileira de Teses e Dissertações (BDTD), nos quais foram encontrados os demais trabalhos (49).

Observa-se que a produção anual de teses e dissertações começa a ser mais expressiva a partir de 2008, sendo que o número de trabalhos defendidos entre 2008 e 2013 representa 92\% (83) do total (Figura 3). Nota-se também queda na produção em 2013, que pode ser atribuída ao processo moroso de envio das informações aos bancos de dados considerados e ao processo de inserção e reestruturação do Banco da CAPES.

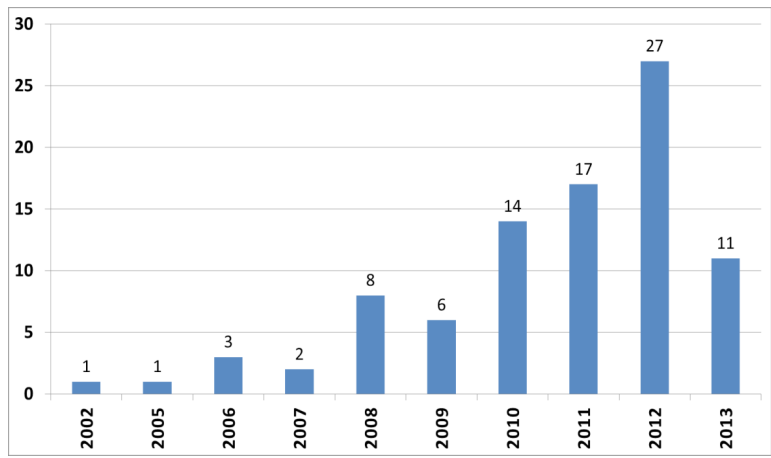

FIGURA 3 - Distribuição das teses e dissertações sobre desastres no Brasil, no período de 2002 a 2013.

FONTE: Elaboração das autoras.

Quanto às áreas de conhecimento, verifica-se que Ciências Ambientais tem o maior número de trabalhos (13\%), seguida por Engenharia Sanitária (11\%), Geografia (10\%) e Psicologia (9\%), que juntas perfazem $43 \%$ do total (Tabela 2). Se consideradas as grandes áreas, destaca-se Engenharias, que agrupadas contribuem com $20 \%$ do total (19).

TABELA 2 - Distribuição dos trabalhos brasileiros sobre desastres, defendidos no período de 2002 a 2013, por área de conhecimento da CAPES.

\begin{tabular}{lcc}
\hline \multicolumn{1}{c}{ Área do Conhecimento (Básica $\mathbf{- ~ 2}^{\mathbf{0}}$ nível - CAPES) } & $\mathbf{N}^{\mathbf{0}}$ Teses + Dissertações & $\%$ \\
\hline Ciências ambientais & 12 & 13 \\
Engenharia sanitária & 10 & 11 \\
Geografia & 9 & 10 \\
Psicologia & 8 & 9 \\
Planejamento urbano e regional & 7 & 8 \\
Sociologia & 7 & 8 \\
Engenharia civil & 6 & 7 \\
Geociências & 5 & 6 \\
Interdisciplinar & 5 & 6 \\
Direito & 4 & 4 \\
Serviço social & 4 & 4 \\
\hline
\end{tabular}




\begin{tabular}{lcc}
\hline \multicolumn{1}{c}{ Área do Conhecimento (Básica - $\mathbf{2}^{\mathbf{0}}$ nível - CAPES) } & $\mathbf{N}^{\mathbf{0}}$ Teses $^{-}$Dissertações & $\%$ \\
\hline Arquitetura e urbanismo & 2 & 2 \\
Antropologia & 1 & 1 \\
Ciência política & 1 & 1 \\
Comunicação & 1 & 1 \\
Educação & 1 & 1 \\
Enfermagem & 1 & 1 \\
Engenharia de produção & 1 & 1 \\
Engenharia de transportes & 1 & 1 \\
Engenharia elétrica & 1 & 1 \\
História & 1 & 1 \\
Medicina & 1 & 1 \\
Saúde coletiva & 1 & 1 \\
TOTAL & $\mathbf{9 0}$ & $\mathbf{1 0 0}$ \\
\hline
\end{tabular}

FONTE: Elaboração das autoras.

A leitura dos resumos desses trabalhos permitiu identificar que 37 (cerca de 40\%) estão relacionados a impactos decorrentes de eventos de chuvas, como inundações, enchentes e escorregamentos.

Nos 90 trabalhos encontrados foram identificadas 144 diferentes palavras-chave, das quais 132 foram utilizadas no máximo em dois trabalhos. Excluindo-se as palavras "desastre" ou "desastres", utilizadas para o levantamento inicial, dentre as três palavras-chave mais utilizadas encontram-se "risco", "vulnerabilidade" e "defesa civil", presentes respectivamente em 32, 20 e 14 trabalhos. Observa-se ainda a utilização de outras quatro palavras-chave associadas à incidência de chuvas (chuva(s), inundação, enchente(s) e escorregamento(s)), que totalizam 22 ocorrências.

\subsection{Artigos em periódicos cientificos brasileiros da base de dados SCIELO}

Foram encontrados 27 artigos na base de dados SciELO (Figura 3), cerca de 90\% (25) deles publicados a partir de 2008; o número mais expressivo de publicações anuais registrado em 2012 e 2013 indica que o tema tem evoluído como objeto de estudo.

As publicações concentraram-se em três áreas de conhecimento (63\%): Psicologia, Geociências e Sociologia (Tabela 3). No geral, 23 artigos abordaram algum tipo de desastre natural, dois se referiram ao fenômeno desastre de forma genérica e dois abordaram especificamente desastres aéreos.

No total foram utilizadas 83 palavras-chave diferentes, 71 empregadas uma única vez. As palavras-chave mais utilizadas foram "desastre natural", "desastre" e "saúde da família", que ocorreram respectivamente em 15, 6 e 3 artigos. As palavras-chave mais utilizadas no levantamento das teses e dissertações, "vulnerabilidade", "risco" e "defesa civil", aparecem aqui em duas publicações apenas. Outras palavras-chave que também ocorrem em pelo menos duas publicações são: “enchente(s)", "desastre aéreo", "comunidade(s)", "enfermagem", "profissionais", "terremoto" e "transtorno de estresse pós-traumático". 
TABELA 3 - Distribuição dos artigos sobre desastres publicados em periódicos científicos brasileiros, no período de 2001 a 2013, por área de conhecimento da CAPES.

\begin{tabular}{lcc}
\hline $\begin{array}{c}\text { Áreas do } \\
\text { Conhecimento }\end{array}$ & $\begin{array}{c}\text { Número de } \\
\text { Publicações (n) }\end{array}$ & $\begin{array}{c}\text { Porcentagem de } \\
\text { Publicações (\%) }\end{array}$ \\
\hline Psicologia & 9 & 33 \\
Geociências & 4 & 15 \\
Sociologia & 4 & 15 \\
Saúde Coletiva & 3 & 11 \\
Enfermagem & 3 & 11 \\
Ciências Ambientais & 2 & 7 \\
Serviço Social & 1 & 4 \\
Comunicação & 1 & 4 \\
Total & $\mathbf{2 7}$ & $\mathbf{1 0 0}$ \\
\hline
\end{tabular}

FONTE: Elaboração das autoras.

\subsection{Artigos em periódicos internacionais da base de dados WoS}

Das 13 publicações em periódicos internacionais selecionadas de acordo com o método proposto, no período de 2000 a 2013, uma se refere a desastres em geral, abordando a questão dos serviços farmacêuticos; oito abordam ocorrências de desastres naturais; e três versam sobre desastres tecnológicos. A área de Saúde Pública, Ambiental e Ocupacional apresenta maior número de artigos (4). Observa-se a publicação de um único trabalho no ano 2000 , e a partir de 2008 verifica-se uma produção de dois artigos a cada dois anos (2008, 2010 e 2012), culminando com a publicação de seis artigos em 2013, conforme Figura 1. A distribuição dessas publicações segundo as áreas de pesquisa a que se referem, de acordo com a Web of Science (WoS), está apresentada na Tabela 4.
TABELA 4 - Distribuição dos artigos sobre desastres publicados em periódicos internacionais, no período de 2000 a 2013, por área de pesquisa da base de dados WoS.

\begin{tabular}{lcc}
\hline \multicolumn{1}{c}{$\begin{array}{c}\text { Área de } \\
\text { Pesquisa (WoS) }\end{array}$} & $\begin{array}{c}\mathbf{N}^{\mathbf{0}} \\
\text { Publicações } \\
\text { (n) }\end{array}$ & $\begin{array}{c}\text { Percentual } \\
\text { de Publicações } \\
\text { (\%) }\end{array}$ \\
\hline $\begin{array}{l}\text { Saúde Pública } \\
\text { Ambiental e } \\
\begin{array}{l}\text { Ocupacional } \\
\text { Meteorologia e }\end{array}\end{array}$ & 4 & 31 \\
Ciências Atmosféricas & 2 & 15 \\
Geografia & 2 & 15 \\
Ciências Ambientais & 2 & 15 \\
Administração Pública & 1 & 8 \\
Estudos Urbanos & 1 & 8 \\
Florestal & 1 & 8 \\
Total & $\mathbf{1 3}$ & $\mathbf{1 0 0}$ \\
\hline
\end{tabular}

FONTE: Elaboração das autoras.

\section{Discussão}

Os resultados indicam as principais áreas do conhecimento que têm se dedicado à realização de pesquisas sobre o tema, à produção acadêmica em formato de teses e dissertações e as publicações em periódicos nacionais e internacionais, com autores e/ou coautores brasileiros. Observa-se que a produção científica tanto em periódicos quanto em teses e dissertações tem um incremento significativo a partir de 2008, e que, em sua maioria, está direcionada a desastres naturais. Uma de nossas hipóteses é que esse adensamento na produção científica pode estar relacionado ao debate já corrente sobre desastres ambientais, às ocorrências mais frequentes de eventos climáticos extremos no país e à divulgação, 
em 2007, do $4^{\circ}$ Relatório do Painel Intergovernamental sobre Mudanças Climáticas (IPCC, 2007).

$\mathrm{O} 4^{\circ}$ relatório do IPCC teve grande repercussão mundial nos meios de comunicação, governos, comunidade científica e sociedade. Nesse documento, a magnitude dos impactos das mudanças climáticas foi avaliada com mais critério, devido à maior quantidade de informações disponíveis, incluindo regiões não cobertas nas avaliações anteriores. Ainda, as principais conclusões do documento associadas à ocorrência de desastres revelaram que: i) a temperatura mundial poderia aumentar entre 1,1 e $6,4^{\circ} \mathrm{C}$, durante o século XXI; ii) o nível do mar provavelmente se elevaria entre 18 e 59 cm; iii) são esperadas ocorrências de maior derretimento glacial, ondas de calor e chuvas torrenciais; e iv) a possibilidade de aumento das secas, ciclones tropicais e marés altas elevadas.

Por outro lado, no mesmo ano de divulgação do relatório do IPCC, no contexto do Plano de Ação de Ciência, Tecnologia e Inovação para o Desenvolvimento Nacional, foi instituída a Rede Brasileira de Pesquisas sobre Mudanças Climáticas Globais (Rede Clima), por meio da Portaria $n^{\circ}$ 728/2007 do Ministério da Ciência e Tecnologia (MCT). Ainda como repercussão da divulgação do relatório, em 2009, no Brasil, foi instituído pelos ministérios do Meio Ambiente e da Ciência e Tecnologia o Painel Brasileiro sobre Mudanças do Clima. Nessa perspectiva, a Fundação de Amparo à Pesquisa do Estado de São Paulo (FAPESP) lançou, em 2008, o Programa FAPESP de Pesquisa em Mudanças Climáticas Globais (PFPMCG), com abertura do primeiro edital de chamada de propostas de pesquisas no mesmo ano. Todas essas medidas podem, de certa forma, ter contribuído para o incremento da produção científica com foco na temática dos desastres.

Os resultados revelam também que há predominância de grupos de pesquisa das áreas de Geociências e Engenharias. Nesse levantamento, foram encontrados apenas três grupos de pesquisa do $\mathrm{CNPq}$ das áreas de Ciências Humanas (Geografia). Quanto às linhas de pesquisa com o termo "desastre" na identificação, pertencentes a outros grupos de pesquisa, seis são da área Ciências Sociais Aplicadas e sete de Ciências Humanas. Além disso, uma leitura dos editais lançados no período parece indicar maior ênfase às áreas temáticas das Exa- tas. Consideramos que o fortalecimento e a ampliação da produção científica nas áreas de Ciências Humanas demandam também o reconhecimento das agências de fomento por meio de seus editais.

Dos artigos publicados em periódicos brasileiros, as áreas de Psicologia, Geociências e Sociologia são predominantes, enquanto naqueles publicados em periódicos internacionais há um destaque para a área classificada na WoS como Saúde Pública, Ambiental e Ocupacional (Public Environmental Occupational Health).

Deve-se observar que os resultados encontrados, a partir das palavras-chave selecionadas, limitaram-se à pesquisa científica diretamente voltada aos desastres, não englobando trabalhos com foco em aspectos particulares dos eventos.

Lembramos que a terminologia e os critérios quantitativos utilizados na área de pesquisa e gerenciamento de desastres, que consideram principalmente número de mortos e feridos, afetados e perdas econômicas para sua caracterização, não são padronizados e carecem de maior clareza e consenso - vale lembrar a discrepância dos números encontrados em fontes distintas, como é o caso do Atlas Brasileiro de Desastres Naturais - 1991 a 2010 e do EM-DAT. Cabe ainda questionar se os critérios adotados para a conceituação de desastre não mereceriam uma perspectiva mais interdisciplinar para a construção do conhecimento nesse campo.

Mesmo considerando essas limitações, quando se compara o número encontrado de 5,4 mil publicações em periódicos internacionais com as mesmas palavras-chave, obtido em pesquisa na base de dados WoS, com as 27 publicações encontradas na base de dados SciELO Brasil, o presente levantamento indica que o campo de pesquisas sobre desastres no Brasil necessita ser ampliado em todas as áreas de conhecimento.

Reconhece-se o direcionamento e a priorização de pesquisas sobre desastres naturais; contudo, considerando que a sociedade contemporânea é caracterizada pela concentração e pela interdependência de pessoas, da produção e de serviços em áreas urbanas, altamente adensadas, argumenta-se que outros tipos de desastres devam ser incluídos como objeto de estudo, como os decorrentes de origem antropogênica de natureza tecnológica, social ou biológica. 
Como sugestão de estudos que poderiam ser desenvolvidos na temática dos desastres, apresenta-se a seguir um rol de possibilidades:

- Pesquisa e sistematização de práticas na prevenção de desastres para constituição de sistemas de informação e comunicação interativos;

- Identificação e construção das capacidades de prevenção de desastres e dos recursos necessários e disponíveis para essa finalidade;

- Estudos para o estabelecimento de critérios científicos para elaboração de alertas de desastres naturais no país;

- Desenvolvimento de conceitos e instrumentos para avaliação das práticas utilizadas na prevenção dos diversos tipos de desastres;

- Identificação de fatores de resiliência das comunidades em face dos desastres, com a definição de indicadores;

\section{Referências}

BDTD - Biblioteca Digital Brasileira de Teses e Dissertações Disponível em <http://bdtd.ibict.br/>. Acesso em: 12 fev. 2014.

Below, R.; Wirtz, A.; Sapir, D. G. Disaster category classification and peril terminology for operational purposes (2009) (Working paper). CRED - Centre for Research on the Epidemiology of Disasters. Disponível em: $<$ http://www. emdat.be/publications>. Acesso em: 12 fev. 2014.

Brasil. Lei 12.608, de 10 de abril de 2012a, que institui a Política Nacional de Proteção e Defesa Civil - PNPDEC. Brasília: DOU de 11/4/2012.

Brasil. Ministério da Integração Nacional. Instrução Normativa n. 1, de 24 de agosto de 2012b. Diário Oficial da União. Seção 1, n. 169, quinta-feira, 30 de agosto de 2012 (b). Disponível em: $<$ http://www.in.gov.br/visualiza/index.jsp?data $=30 / 08 /$ $2012 \&$ jornal $=1 \&$ pagina $=30 \&$ total Arquivos $=120>$. Acesso em: 20 fev. 2014

Carmo, R. L. do. Urbanização e desastres: desafios para a segurança humana no Brasil. In: Carmo, R.L.; Valêncio, N. (Orgs.). Segurança humana no contexto dos desastres. São Carlos: RiMa, 2014.
- Desenvolvimento de métodos integrados e transversais para elaboração, implantação e avaliação de ações e políticas públicas;

- Estudos voltados às ações de saneamento ambiental e gestão sustentável de resíduos pós-desastres, visando à redução de impactos ambientais e riscos à saúde humana.

Reconhece-se que o levantamento realizado não contempla a totalidade da produção científica nacional, que pode estar dispersa em outros tipos de publicações de acesso mais restrito como: artigos em revistas não indexadas, relatórios de pesquisa, trabalhos publicados em anais de encontros científicos, livros, capítulos de livros, trabalhos de conclusão de curso. Entretanto, a intenção do recorte adotado pelas autoras foi contribuir para o debate sobre o tema e revelar as tendências, a partir de duas bases de dados de caráter multidisciplinar e amplo acesso, complementadas por pesquisas desenvolvidas nos níveis de mestrado e doutorado, materializadas na produção de teses e dissertações.

CAPES - Coordenação de Aperfeiçoamento de Pessoal de Nível Superior. Banco de Teses e Dissertações. Disponível em: <http://bancodeteses.capes.gov.br/>. Acesso em: 30 mar. 2014.

CEPED- UFSC. Centro Universitário de Estudos e Pesquisas sobre Desastres da Universidade Federal de Santa Catarina. Atlas brasileiro de desastres naturais 1991 a 2010: volume Brasil. Florianópolis: CEPED UFSC, 2012. 94 p.

CNPq - Conselho Nacional de Desenvolvimento Científico e Tecnológico. Diretório de grupos de pesquisa no Brasil: Consulta Parametrizada. Disponível em: < http://dgp.cnpq. $\mathrm{br} / \mathrm{dgp} /$ faces/consulta/consulta_parametrizada.jsf $>$. Acesso em: 29 out. 2014.

EM-DAT. Emergency Events Database. Disponível em: <http:// www.emdat.be/database>. Acesso em: 4 abr. 2014.

Freitas, C. M.; Carvalho, M. L. de; Ximenes, E. F.; Arraes, E. F.; Gomes, J. O. Vulnerabilidade socioambiental, redução de riscos de desastres e construção da resiliência: lições do terremoto no Haiti e das chuvas fortes na Região Serrana, Brasil. Ciência e Saúde Coletiva, 17(6), 1577-1586, 2012. 
GRIP - Global Risk Identification Programme. Disaster Loss Data Standards. 2008. Disponível em: <http://www.gripweb. org/gripweb/?q=methodologies-tools-portal $>$. Acesso em: 3 abr. 2014.

IPCC - Intergovernmental Panel On Climate Change. Climate Change 2007: Synthesis Report. Disponível em: < http://www. ipcc.ch/pdf/assessment-report/ar4/syr/ar4_syr_sp.pdf $>$. Acesso em 27 fev. 2014.

IPCC - Intergovernmental Panel On Climate Change. Climate Change 2014: Impacts, Adaptation, and Vulnerability. WGII AR5 Chapter 8. Urban Areas, 2014. Disponível em: <http:// www.ipcc.ch/report/ar5/wg2/ HYPERLINK "http://www.ipcc. ch/report/ar5/wg2/".> Acesso em: 10 abr. 2014.

ISDR - International Strategy for Disaster Reduction. Terminology. 2009. Disponível em: <http://www.unisdr.org/ files/7817_UNISDRTerminologyEnglish.pdf $>$. Acesso em: 20 jan. $20 \overline{14}$

Kendra, J.; Knowles, S. G.; Wachtendorf, T. 2011 Annual Report. Disaster Research Center (DRC) Research Office University of Delaware. Disponível em: <http://www.udel.edu/DRC/ aboutus/AnnualReports/2011.pdf> Acesso em: 20 jan. 2014.

Lindell, M. K. Disaster studies. Sociopedia.isa, 1-18, 2011. doi: $10.1177 / 205684601111$.

Martins, H. Experimentum Humanum: Civilização tecnológica e condição humana. Lisboa: Relógio D’Água, 2011.

Portal Domínio Público. Disponível em: <http://www.dominiopublico.gov.br/>. Acesso em: 05. abr. 2014.

Ribeiro, M. J. Sociologia dos desastres. Sociologia, Problemas e Práticas, 18, 23-43, 1995.

SciELO Brasil. Disponível em: <http://www.scielo.br/>. Acesso em: 20 jun. 2014.
Soriano, E; Coutinho, M. P.; Londe, L. R; Saito, S. M.; Gregorio, L. T. Avaliação de propostas de candidatos a prefeitos em relação aos riscos de desastres naturais. Sociedade \& Natureza, 25(3), 525-542, 2013.

Tominaga L. K.; Santoro, J.; Amaral, R. Desastres naturais: conhecer para prevenir. São Paulo: Instituto Geológico, 2009.

United Nations. World Urbanization Prospects: The 2012 Revision. UN Department of Economic and Social Affairs, Population Division, 2012. Disponível em: < World Urbanization Prospects: The 2012 Revision>. Acesso em: 30 nov. 2013.

UNISDR - United Nations Office for Disaster Risk Reduction. Hyogo Framework for Action 2005-2015: Building the resilience of nations and communities to disasters. summary, 2005. Disponível em: $<$ http://www.unisdr.org/files/1037 finalreportwcdrspanish1.pdf $>$. Acesso em: 30 mar. 2014.

Valêncio, N. Introdução. In: Valencio, M.; Siena, M.; Marchezini, V.; Gonçalves, J. C. (Org.). Sociologia dos desastres: construção, interfaces e perspectivas no Brasil. São Carlos: RiMa, 2009.

Valêncio, N. Desastres, ordem social e planejamento em defesa civil: o contexto brasileiro. Saúde e Sociedade, 19(4), 748-762, 2010.

Valêncio, N. F. L.S. Desastres: tecnicismo e sofrimento social. Ciência \& Saúde Coletiva, 19(9), 3631-3644, 2014.

Web of Science (WoS). Disponível em: $<$ http://apps.webofknowledge.com/WOS_GeneralSearch_input.do?highlighted_ta$\mathrm{b}=$ WOS\&product $=\overline{\text { WOS}} \&$ last_prod=WOS\&search $\_$mode $=-$ GeneralSearch>. Acesso em: 14 mar. 2014.

Wirtz A.; Kron, W.; Löw, P.; Steuer, M. The need for data: natural disasters and the challenges of database management. Natural Hazards, 70, 135-157, 2014. 